\title{
Nanomedicine for glaucoma: liposomes provide sustained release of latanoprost in the eye
}

This article was published in the following Dove Press journal:

International Journal of Nanomedicine

4 January 2012

Number of times this article has been viewed

\author{
Jayaganesh V Natarajan ${ }^{*}$ \\ Marcus Ang2* \\ Anastasia Darwitan' \\ Sujay Chattopadhyay ${ }^{3}$ \\ Tina T Wong ${ }^{2}$ \\ Subbu S Venkatraman
}

'Materials Science and Engineering, Nanyang Technological University, Singapore; ${ }^{2}$ Singapore Eye Research Institute, Singapore; ${ }^{3}$ Polymer Division, Indian Institute of Technology

Roorkee, India

*These authors contributed equally to this work
Correspondence: Subbu S Venkatraman Materials Science and Engineering,

Nanyang Technological University,

Singapore 639798

$\mathrm{Tel}+6567904259$

Email assubbu@ntu.edu.sg

Tina TWong

Singapore Eye Research Institute,

Singapore I6875 I

Tel + 6563228313

Email tina.wong.t.l@snec.com.sg
Purpose: To report the development and therapeutic evaluation of a liposomal nanocarrier for sustained release of latanoprost, in the rabbit eye.

Methods: We fabricated latanoprost-loaded egg-phosphatidylcholine (EggPC) liposomes using the film hydration technique. The delivery vehicles were nano-sized ( $\mathrm{Z}$ avg $=109 \pm 18 \mathrm{~nm})$, had a narrow poly dispersity index (PDI $=0.19 \pm 0.04$ ), and a very high loading efficiency $(94 \% \pm 5 \%)$. Based on in vitro data, we evaluated this formulation for lowering intraocular pressure (IOP) in rabbit eyes. Following a single subconjunctival injection of the latanoprost loaded formulation, the eyes were clinically monitored and the IOP recorded.

Results: Latanoprost-loaded EggPC liposomes demonstrated a high drug/lipid mole ratio of 0.181 , remained stable for at least 6 months on storage $\left(4^{\circ} \mathrm{C}\right)$, and at least 1 month at $25^{\circ} \mathrm{C}$. A slow and sustained release of $60 \%$ of latanoprost was achieved by 14 days in the in vitro release study. The same formulation demonstrated a greater sustained IOP lowering effect compared with daily administration of topical latanoprost beyond 90 days $(4.8 \pm 1.5$ vs $2.5 \pm 0.9 \mathrm{mmHg} ; P<0.001)$. No signs of inflammation were evident in the eyes from slit-lamp examination analysis.

Conclusion: The loading required for a long-term sustained delivery of latanoprost for up to 90 days in the rabbit eyes was achieved with EggPC liposomes. A single injection of latanoprostloaded EggPC liposomes can lower the IOP for up to 90 days, with a greater IOP lowering effect than daily topical administration of latanoprost.

Keywords: nanomedicine, nanoliposomes, EggPC, latanoprost, sustained ocular delivery, glaucoma

\section{Introduction}

Intraocular pressure (IOP) remains the key modifiable risk factor in glaucoma a progressive optic neuropathy resulting in irreversible visual field loss that affects more than 60 million worldwide and is the second leading cause of blindness after cataract. ${ }^{1}$ The first line treatment of glaucoma is topical medications to lower the IOP, thereby delaying damage to the optic nerve from elevated IOP. However, daily application, due to poor ocular bioavailability and other long-term side effects such as allergy and intolerance to medications, have negative effects on patient compliance, which leads to disease progression from suboptimal medical management of the disease resulting in poor IOP control..$^{2-4}$ Latanoprost, a lipophilic drug usually delivered in the form of an oil/water emulsion (Xalatan ${ }^{\circledR}$, Pfizer, Catalent Pharma Solutions, Woodstock, IL), is very effective in reducing IOP in glaucoma. ${ }^{5}$ However, latanoprost acid, the hydrolyzed active product, is more hydrophilic and experiences a higher penetration resistance through the epithelium and endothelium of the cornea and hence lower bioavailability. ${ }^{6}$ 
Liposomes, which have been shown to be biocompatible nanocarriers for ocular use, allows for delivery of both the lipophilic drug molecule as well as its hydrophilic active products, due to its physical structure of a polar core and lipophilic bilayer. ${ }^{7}$ Liposomal encapsulation protects drug molecules from enzymatic hydrolysis in the physiological environment while in circulation, and thus increases stability. Previous studies on topical application of liposomes demonstrated poor penetration into the eye, ${ }^{8-11}$ however, while studies on subconjunctival injections on other IOP-lowering drugs showed limited sustainability., ${ }^{911,12}$ Furthermore, it is not clearly understood whether drug-loaded liposomes in the subconjunctiva behave like a depot system or actually undergo circulation and endocytosis. ${ }^{12}$ The design of carriers for sustained delivery in the eye necessitates the use of a formulation that has the required optical clarity with sufficient drug loading efficiency for administration. Also, drug-loaded carriers should be stable under testing conditions in vitro and in vivo. The size of the nanocarriers might also facilitate the delivery of the drug through the various anatomical structures of the eye (conjunctiva and sclera) to reach the targeted site (ciliary body) more efficiently, with increased bioavailability.

Thus the challenge is to formulate latanoprost into a liposomal vehicle such that its delivery is sustained over several weeks to months, after a single subconjunctival injection. In this study, we developed and optimized a liposomal formulation that provides prolonged delivery, and confirmed its safety and efficacy in the rabbit eye.

\section{Materials and methods Materials}

Egg-phosphatidylcholine (EggPC $>95 \mathrm{wt} \%$ ) was purchased from NOF Corporation (Tokyo, Japan). Polycarbonate filter membranes of sizes $(0.2,0.1$, and $0.08 \mu \mathrm{m})$ and drain discs were purchased from Northern Lipids Inc (Burnaby, Canada). Cellulose ester dialysis bags ( $16 \mathrm{~mm}$ dia $\times 10 \mathrm{~m}$ flat width) were obtained from Spectrum Laboratories (Rancho Dominguez, CA). Chemicals for phospholipid analysis such as ammonium thiocyanate and ferric chloride hexahydrate were purchased from Sigma (Ronkonkoma, NY). Sodium phosphate dibasic anhydrous $\left(\mathrm{Na}_{2} \mathrm{HPO}_{4}\right)$, potassium phosphate monobasic anhydrous $\left(\mathrm{KH}_{2} \mathrm{PO}_{4}\right)$, potassium chloride $(\mathrm{KCl})$, and sodium chloride $(\mathrm{NaCl})$ salts were purchased from Sigma and used without further purification. Latanoprost was purchased from Sinophram Chemical Reagent Co Ltd, Senyang, China. The water used in all the experiments was from MiliQ purification system with a resistivity of at least $18.2 \pm 0.2 \mathrm{~m} \Omega \mathrm{cm}$.
All solvents were purchased from Tedia (Fairfield, $\mathrm{OH}$ ), and all solvent drug estimation was conducted using high performance liquid chromatography (HPLC) grade ( $>99 \%$ purity).

\section{Methods}

\section{Preparation of large unilamellar vesicles}

A thin film hydration technique was used to formulate latanoprost loaded EggPC liposomes as described elsewhere. ${ }^{12}$ Briefly, known amounts of phospholipid EggPC (lipid concentration, $18 \mathrm{mM}$ ) were weighed and dissolved in chloroform:methanol (2:1, vol/vol) solvent mixture. A known volume of the drug solution of latanoprost ( $2 \mathrm{mg} / \mathrm{mL}$ stock solution in acetonitrile) was added to the lipid solvent mixture maintained at $40^{\circ} \mathrm{C}$. An initial drug:lipid mole ratio of 0.154 was used. The solvent mixture was removed from the round bottom flask using a rotary evaporator $\left(\mathrm{IKA}^{\circledR} \mathrm{RV} 10\right.$, IKA ${ }^{\circledR}$ Werke GmbH \& Co. KG, Staufen, Germany) connected to a water bath (IKA ${ }^{\circledR}$ MB 10 basic, IKA ${ }^{\circledR}$ Werke GmbH \& Co. $\mathrm{KG}$, Germany) maintained at $40^{\circ} \mathrm{C}$. To completely eliminate any trace of residual solvent, the flask was rotated at $100 \mathrm{rpm}$, under low pressure for an hour. A thin, drug-loaded, lipid film was obtained and to this film, isotonic phosphate-buffered saline (PBS) (150 mM, pH 5.5) buffer was added to form multilamellar vesicles (MLVs). Subsequent size reductions were carried out by sequential extrusion of MLVs (10 times) through polycarbonate filters of size $(0.2 \mu \mathrm{m} / 0.08 \mu \mathrm{m})$, fitted in a bench top extruder, purchased from Northern Lipids Inc, Canada. At the end of this step, large unilamellar vesicles (LUVs) with a size distribution of $0.09-0.12 \mu \mathrm{m}$ were obtained. All of the above-mentioned steps were performed under aseptic conditions. All glassware and the PBS solution (filtered through $0.2 \mu \mathrm{m}$ filter), was sterilized by autoclaving, and the entire procedure was performed under a laminar flow hood (ESCO, Singapore).

\section{Characterization of drug-loaded EggPC liposomes \\ Size}

The average size of the liposomes as well as the size distribution (poly dispersity index) was carried out using the Malvern Zetasizer Nano ZS (Worcestershire, UK). The vesicle sizes were continuously monitored after preparation, on storage $\left(4^{\circ} \mathrm{C}\right.$ and $\left.25^{\circ} \mathrm{C}\right)$, and after drug release in vitro.

\section{Lipid analysis}

The phospholipid concentration was estimated using colorimetric method as described elsewhere. ${ }^{13} \mathrm{~A}$ six point calibration was prepared for EggPC lipids (peak wavelength 
absorbance at $470 \mathrm{~nm}$ ) and was later used for estimation of the concentration of lipids in vesicles obtained after extrusion. The amount of phospholipids after extrusion was estimated to be around $80 \%-85 \%$ (by mass).

\section{Estimation of drug concentration using high performance liquid chromatography method}

The concentration of the latanoprost was estimated using an HPLC system (Agilent series 1200, Santa Clara, CA). The chromatographic separation was performed on reverse phase Eclipse-XDB C18 column $(5 \mu \mathrm{m}, 4.6 \mathrm{~mm}$ ID $\times 250 \mathrm{~mm})$ using mobile phase as acetonitrile $(\mathrm{ACN})$ :Water at 70:30 $(\mathrm{v} / \mathrm{v})$ proportions. The flow rate was maintained at $1.0 \mathrm{~mL} /$ min and wavelength was set at $210 \mathrm{~nm}$. The retention time was $4.3 \mathrm{~min}$ and the temperature of the column was maintained at $25^{\circ} \mathrm{C}$. The released latanoprost concentration was estimated directly from the collected samples in $\mathrm{pH} 7.4$. The total drug concentration was estimated by breaking the liposomes in a solvent system, such as isopropyl alcohol in a volume ratio of 1:4. The broken lipid mass was then centrifuged and isolated from the rest of solution by ultracentrifugation at 13,000 rpm for 30 minutes. The supernatant was diluted 50 times with PBS pH 7.4 and analyzed for total drug concentration. The total and released drug values were estimated in comparison with a standard calibration curve of latanoprost in PBS pH 7.4. The drug loading efficiency was calculated as follows:

Drug loading efficiency $(\%)=100 \times($ Amount of drug retained /Amount of drug taken initially)

\section{Drug partition coefficient estimation}

A lipophilic drug such as latanoprost distributes between a lipid bilayer and the aqueous continuous phase, and the concentration ratio between these two phases determines the partition coefficient. The values for partition coefficient were estimated from the MLVs before the extrusion step. Briefly, known sample volumes were collected in microfuge tubes and centrifuged at $10000 \mathrm{rpm}$ for 30 minutes. Due to the large size of MLVs, in the micron size ranges, we were able to separate out the fractions of the lipid pellet from the clear supernatant. The drug estimated from the supernatant is a measure of continuous (buffer) phase drug concentration, while this amount when subtracted from the total drug concentration yields drug partitioned into the bilayer. Thus, the drug partition coefficient (P.C.) in MLVs was estimated using the following expression (each concentration in mass per unit volume):
P.C. $=($ Total amount of drug-amount of drug in buffer $)$ /(amount of drug in buffer)

The final drug:lipid ratios in the liposomal formulation obtained after extrusion in LUVs were estimated as follows:

Final drug to lipid (D/L) mole ratio in LUVs after extrusion $=$ moles of drug left $/$ moles of the lipid

\section{Drug release studies}

A dialysis method was followed to evaluate the release of latanoprost from EggPC liposomes. Briefly, $1 \mathrm{~mL}$ of drugloaded liposomal suspension was taken in a cellulose ester dialysis bag ( $100 \mathrm{kD}$ MWCO, $1.6 \mathrm{~cm}$ dia $\times 6 \mathrm{~cm}$ length) and dialyzed against $40 \mathrm{~mL}$ of PBS pH 7.4. The dialysis bag was continuously agitated in an orbital shaker (Sartorius Certomat $^{\circledR}$, Sartorius Stedim North America Inc, Bohemia NY) maintained at $37^{\circ} \mathrm{C}$ at $50 \mathrm{rpm}$. To maintain dynamic sink conditions, similar to in vivo, the dialysate was completely exchanged with fresh PBS pH 7.4 buffer every 24 hours. Aliquots were withdrawn from the release medium and were filtered through a $0.2 \mu \mathrm{m}$ syringe filter and assayed for the released drug. The volume of liposomes and sizes of vesicle in the dialysis tube were recorded at the end of release study to check for any dilution effect and/or size change of vesicles.

\section{Estimation of latanoprost in ocular fluids (aqueous and vitreous humor)}

The aspirated aqueous and vitreous humors were collected in sterile $1 \mathrm{~mL}$ syringes. The humors were directly filtered through $0.2 \mu \mathrm{m}$ sterile syringe filters (regenerated cellulose) into microfuge tubes. The samples in the microfuge tubes were then vortexed and ultra-centrifuged at 13,000 rpm for 15 minutes to remove any particulates/debris. The supernatant was then aspirated into a syringe and transferred into amber colored HPLC vials with insets. The latanoprost amount in these fluids was estimated by the HPLC method as earlier described, using the volume of aqueous humor as $0.15 \mathrm{~mL}$ and vitreous humor as $0.3 \mathrm{~mL}$ from a rabbit eye.

\section{In vivo evaluation in the rabbit eye}

\section{Surgical technique}

Sixteen female New Zealand White rabbits were used in this study. The baseline IOP was measured twice daily using a calibrated Tono-pen ${ }^{\circledR}$ XL (Reichert Ophthalmic Instruments, Depew, NY) for 7 days in all rabbits. The rabbits were divided into two treatment groups: (i) Group A (8 rabbits, 16 eyes) received one subconjunctival injection of latanoprost-loaded liposomes using a 27-gauge needle; (ii) Group B (8 rabbits, 
16 eyes) received one eye drop of topical latanoprost daily. All procedures were performed under topical anesthesia (amethocaine 2.5\%) by a single surgeon (MA). Essentially, the eye was cleaned with $50 \%$ povidone iodine and $0.1 \mathrm{~mL}$ of liposome was injected in the subconjunctival space in the superior temporal region of each eye. Topical chloramphenicol $2.5 \%$ was administered to the operated eye daily for 5 days. We obtained approval from the SingHealth Institute Animal Care and Use Committee and all procedures were performed in accordance with the Association for Research in Vision and Ophthalmology (ARVO) Statement for the Use of Animals in Ophthalmic and Vision Research.

\section{Clinical evaluation, slit-lamp microscopy, anterior segment imaging}

Twice-daily IOP measurements were conducted at the same time everyday using a calibrated Tono-pen, and visual inspection of all eyes after injections, or topical latanoprost for signs of conjunctival irritation, inflammation or infection at the injection site. Slit-lamp microscopic examination of the exterior and anterior chamber of the eyes was performed before the injections and weekly thereafter. Anterior segment photographs and anterior segment optical coherence tomography (AS-OCT) of the eyes that received the liposome formulation were performed at weekly intervals. The rabbits were also monitored for any gross changes such as eye discharge, squinting, or abnormal behavior suggesting pain or severe discomfort. A modified Hackett McDonald ocular score was used to grade conjunctival injection, conjunctival swelling, discharge, corneal clarity, and aqueous flare. ${ }^{14}$

\section{Statistical analysis}

Statistical analysis included descriptive statistics, where the mean and standard deviation (SD) was calculated for the continuous variables; while frequency distribution and percentages were used for categorical variables. Comparisons between categorical variables were conducted by Fisher exact test, whereas the one-way analysis of variance (ANOVA) test was used for means. A $P$ value $<0.05$ was considered statistically significant.

\section{Results Drug-loading efficiency}

The particle sizes of latanoprost loaded EggPC liposomes were in the nanometer range $(\mathrm{Z}$ avg $=109 \pm 18 \mathrm{~nm})$, with a narrow poly dispersity index $(0.19 \pm 0.04)$. A high loading efficiency of latanoprost in EggPC liposomes was achieved $(94 \% \pm 5 \%)$ for an initial D/L mole ratio of 0.154 . The final latanoprost concentration in EggPC liposomes after extrusion was estimated to be greater than $1 \mathrm{mg} / \mathrm{mL}$, which is 20 -fold higher than commercially available topical latanoprost $(50 \mu \mathrm{g} / \mathrm{mL})$ that is administered daily. ${ }^{15}$ The final drug/lipid mole ratio value was estimated to be 0.181 . The concentration of the drug used for in vivo studies was $1.18 \mathrm{mg} / \mathrm{mL}$. The reason for the higher final value of drug/lipid mole ratio was the partial loss of lipids during extrusion.

\section{In vitro size stability}

The changes in the size of the liposomes upon storage as well as during drug release were continuously monitored in a Zetasizer (Malvern Instruments, Malvern, UK) at various temperatures. As shown in Table 1, the unloaded EggPC vesicles showed a significant change in the vesicle size and poly dispersity, compared with latanoprost-loaded EggPC liposomes. The unloaded vesicles aggregated on storage, and micron sized particles were observed within 6 months upon storage at $4^{\circ} \mathrm{C}$. However, the latanoprost-loaded EggPC liposomes were stable with respect to aggregation for at least 6 months on storage at $4^{\circ} \mathrm{C}$ and at least for a month at $25^{\circ} \mathrm{C}$.

\section{In vitro drug release from EggPC vesicles}

The release of latanoprost from the liposomes was evaluated by a dialysis technique and expressed in terms of cumulative drug release (\%) over time as shown in Figure 1. A slow and

Table I Size measurement of liposomes during storage at $4{ }^{\circ} \mathrm{C}, 25^{\circ} \mathrm{C}$ and after drug release in PBS buffer $(\mathrm{pH} 7.4)$ at $37^{\circ} \mathrm{C}$

\begin{tabular}{|c|c|c|c|c|c|c|}
\hline \multirow{5}{*}{$\begin{array}{l}\text { Type of } \\
\text { liposome }\end{array}$} & \multicolumn{6}{|c|}{ Size measurement, nm (PDI) } \\
\hline & \multicolumn{6}{|c|}{ Temperature $\left({ }^{\circ} \mathrm{C}\right)$} \\
\hline & $4^{\circ} \mathrm{C}$ & & & & $25^{\circ} \mathrm{C}$ & $37^{\circ} \mathrm{C}$ \\
\hline & \multicolumn{5}{|c|}{ Duration (months) } & \\
\hline & $\overline{0}$ & I & 2 & 6 & $\mathbf{I}$ & \\
\hline & & & & & & $\begin{array}{l}\text { After in vitro } \\
\text { drug release }\end{array}$ \\
\hline EggPC liposome & $79.8(.09)$ & $114.4(0.19)$ & $180.3(0.34)$ & $1200(0.46)$ & NA & NA \\
\hline Latanoprost loaded & $86.6(0.17)$ & $83.91(0.14)$ & $89.98(0.14)$ & $86.38(0.1 \mathrm{I})$ & $82.88(0.18)$ & $82.02(0.08)$ \\
\hline EggPC liposomes & & & & & & \\
\hline
\end{tabular}

Abbreviations: EggPC, egg-phosphatidylcholine; PBS, phosphate-buffered saline; PDI, poly dispersity index. 


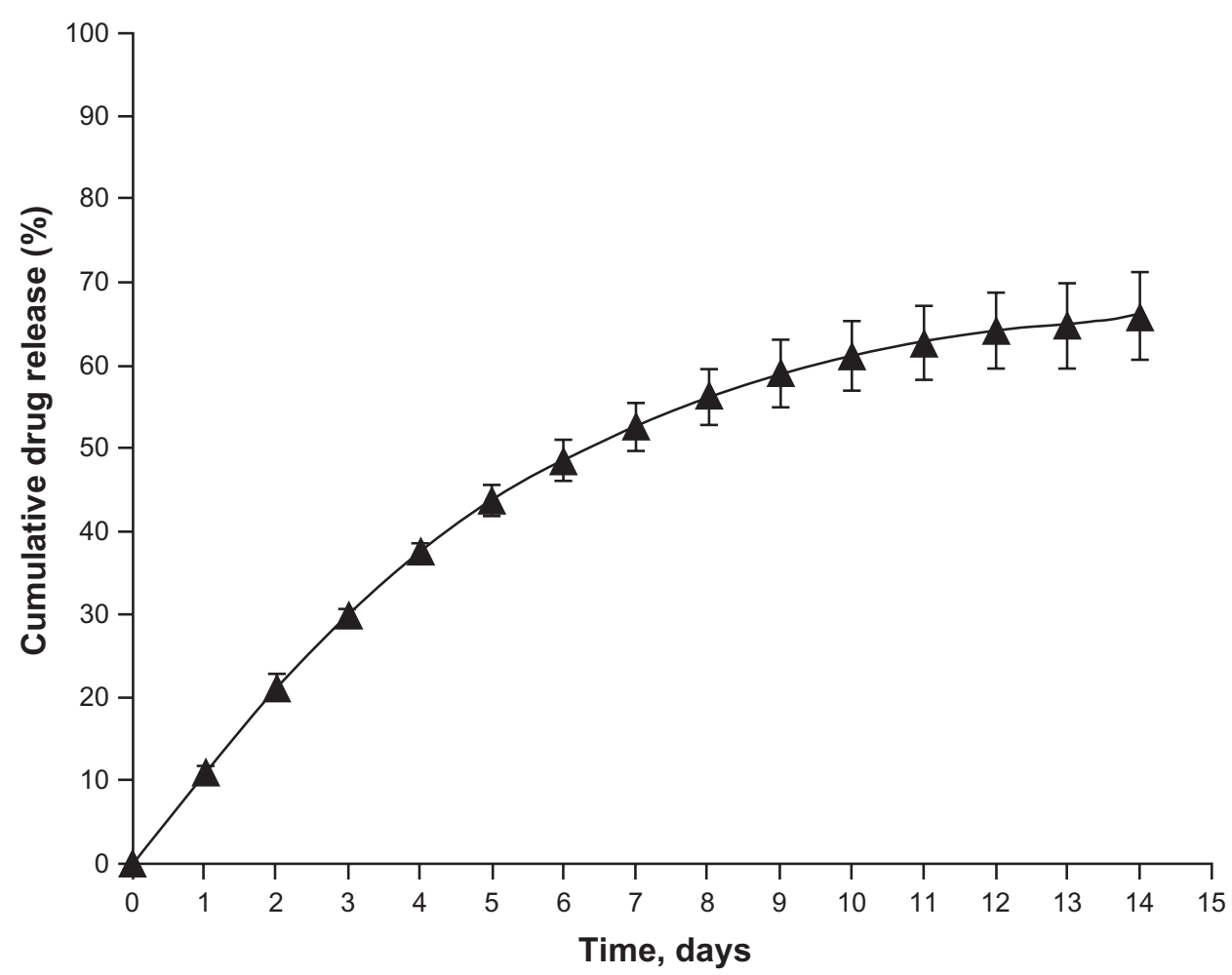

Figure I In vitro dialysis release study of latanoprost from egg-phosphatidylcholine (EggPC) liposomes obtained after extrusion. Cumulative latanoprost release (\%) from EggPC liposomes is plotted against time (days) for a drug/lipid mole ratio of 0.181 .

Notes: Each value is plotted as the average of the results obtained from three independent experiments. The standard deviation is plotted as error bars, which is less than $4 \%$.

sustained release of the drug was achieved with $60 \%$ of the drug being released at the end of 2 weeks. In addition, the size changes were minimal at the end of the in vitro drug release period (Table 1).

Based on the data derived from loading, release, and stability studies, we then investigated the efficacy of IOP lowering from latanoprost loaded-EggPC liposomes (D/L mole ratio of 0.181 ) in rabbit eyes. The dose/day was targeted for at least 2 weeks, similar to that of topical latanoprost ( $1.5 \mu \mathrm{g} / \mathrm{drop} /$ day); to sustain delivery at a level comparable to or higher than the eye drop, about $1 \mathrm{mg} / \mathrm{mL}$ in $100 \mu \mathrm{L}$ of liposomal injection is necessary. ${ }^{16}$ The actual amount of drug released per day from EggPC liposomes was compared to topical latanoprost formulation as shown in Figure 2. The consequences of the higher initial burst seen with liposomes, which would probably be indicated by the presence of a very sudden lowering of the IOP, were carefully monitored in the animals.

\section{Drug partition coefficient value}

The ratio of the drug concentration in the lipid phase to that in the continuous phase determines the partition coefficient (P.C.). A partition coefficient value of $28 \pm 5$ was estimated for EggPC multilamellar liposomes. This value translates to a greater than $95 \%$ of the drug associated/entrapped within the bilayer. The partition coefficient value obtained for EggPC liposomes was significantly higher than observed with dipalmitoylphosphatidylcholine (DPPC) liposomes $(11 \pm 1) .{ }^{16}$ This indicates that a more preferential association of latanoprost was observed with the less ordered liposomal structures derived from EggPC than in a more ordered liposomal structures derived from DPPC.

\section{In vivo study}

Intraocular pressure

The baseline IOP was $13.6 \pm 0.3 \mathrm{mmHg}$ for all 16 rabbits and there was no difference between the two treatment groups $(P=0.80)$. We found a significantly higher mean reduction of IOP in the eyes that received a subconjunctival injection of latanoprost-loaded liposomes compared with eyes administered daily topical latanoprost eye drops. This effect was maintained for 90 days $(4.8 \pm 1.5$ vs $2.5 \pm 0.9 \mathrm{mmHg}$; $P<0.001$ ) (Figure 3). Furthermore, the initial burst release seen in vitro did not appear to result in an excessive IOP reduction $(<2 \mathrm{mmHg})$.

\section{Modified Hackett-Macdonald Scores}

We compared the Modified Hackett-Macdonald Scores of the injection site, by calculating the percent increase in score from pre-injection to the end of the study (90 days) in each 


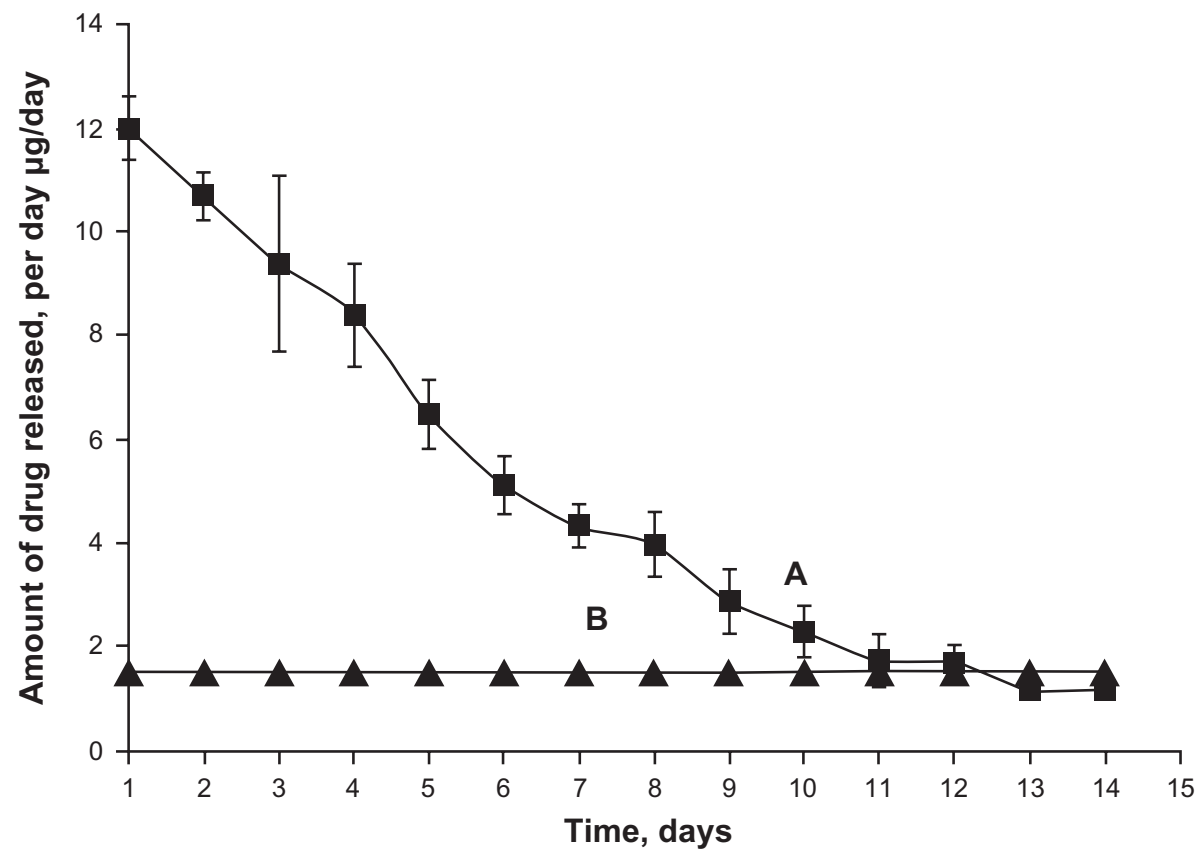

Figure 2 In vitro latanoprost released per day from $100 \mu \mathrm{L}$ of egg-phosphatidylcholine liposomes with a drug/lipid mole ratio of $0.18 \mathrm{I}$ (A) compared with I drop of oncedaily topical latanoprost ( $1.5 \mu \mathrm{g} / \mathrm{drop})$ (B). The amount released per day is reported based on average values of at least two batches and standard deviations are reported in error bars.

treatment group. There was no significant difference in the scores between the two treatment groups $(10 \% \pm 0.5 \%$ vs $12 \% \pm 0.6 \% ; P=0.32$ ). Neither did we observe any clinical complications such as conjunctival infections or anterior chamber inflammation on slit-lamp examination.

\section{Anterior segment optical coherence tomography (AS-OCT)}

AS-OCT scans confirmed subconjunctival location of liposomes in the superior temporal aspect of each rabbit eye immediately after injections as shown in Figure 4.

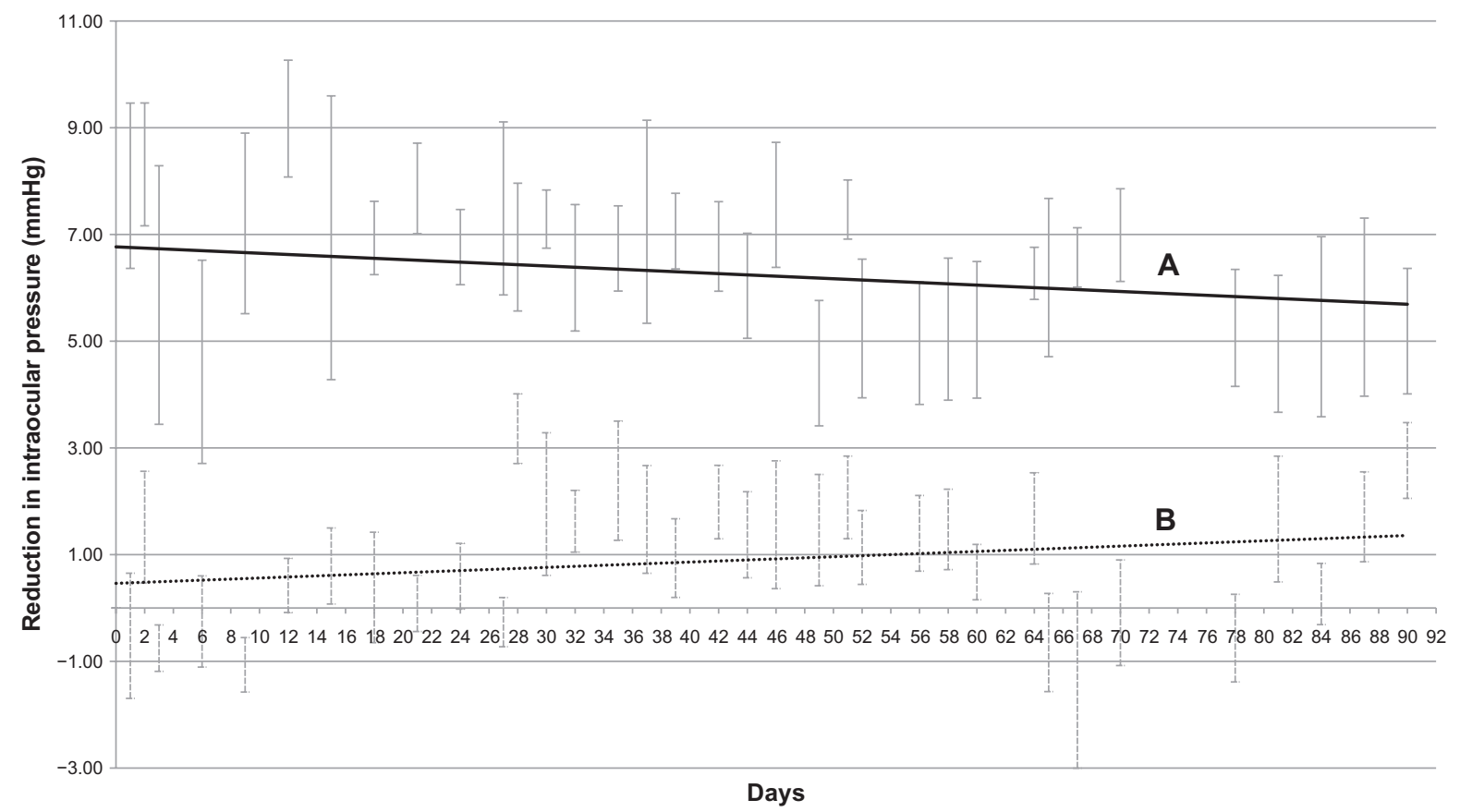

Figure 3 In vivo intraocular pressure (IOP) recordings (reduction in intraocular pressure compared to baseline) comparing the latanoprost loaded liposome group to the control group (once-daily topical latanoprost). (A) Best-fit line for mean IOP reduction in latanoprost-loaded liposome group. (B) Best-fit line for mean IOP reduction in control group. There were significant differences between the two groups, in intraocular pressure readings throughout the 90 -day study period. 


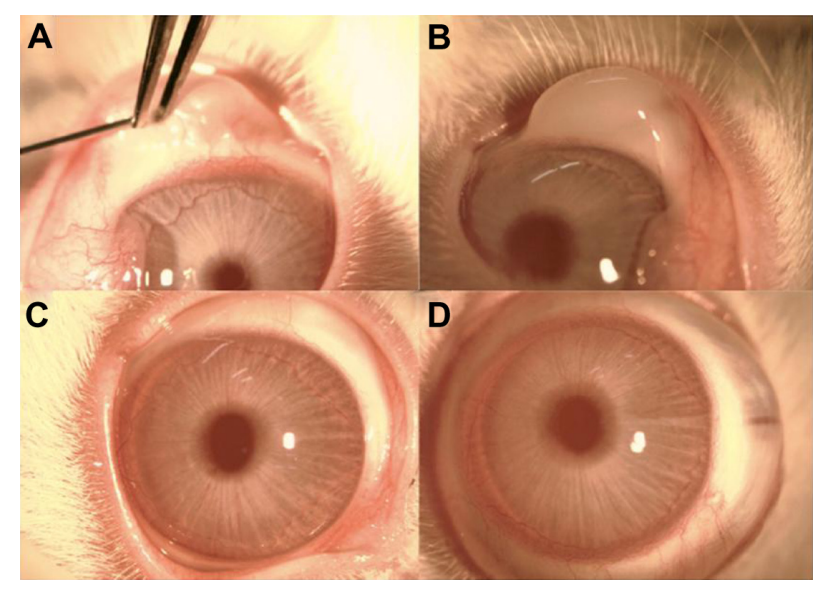

Figure 4 (A) Injection of subconjunctival liposome formulation. (B) Immediate post-injection of liposome formulation. A subconjunctival bleb is clearly seen where the liposomal formulation has been administered. (C) Slit-lamp photograph of eye post-injection at Day 90. The conjunctiva is white and the eye is quiet. There is no evidence of conjunctival hyperaemia, inflammation, or infection at the site of or surrounding the injection area. (D) Slit-lamp photographs of eye receiving one drop of topical latanoprost daily at Day 90.

Subsequent AS-OCT scans performed reveal no abnormal local tissue scarring or scleral thinning at the injection sites as shown in Figure 5. At the end of the experimental period the eyes were enucleated and the ocular fluids examined. There were unrecordable amounts of latanoprost detected in both aqueous and vitreous samples by HPLC analysis from both groups.

\section{Discussion}

Our study revealed that a single subconjunctival injection of latanoprost-loaded EggPC liposomes can effectively lower the IOP in rabbit eyes for at least 90 days. This IOP-lowering effect was significantly greater than once-daily topical

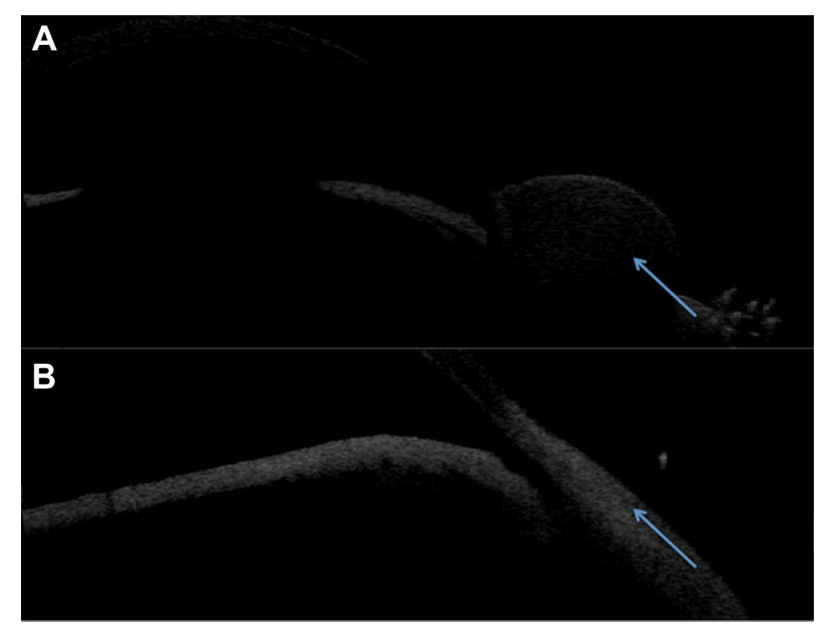

Figure 5 Anterior segment optical coherence tomography confirming the subconjunctival location of the liposome formulation immediate post-injection (A) and no evidence of scleral thinning or conjunctival scarring at Day 90 (B). latanoprost, the current standard treatment in glaucoma patients. These observed results are also consistent with our in vitro results, which demonstrate a slow and sustained release of latanoprost, with stability in liposome size and structural integrity. The sustained reduction of IOP in rabbit eyes is encouraging. To our knowledge, no other reports show such a significant effect of latanoprost sustained-release using liposomes (or any other carriers) in the animal eye. We also demonstrated that the liposome formulation was well tolerated as evidenced from the lack of recorded ocular irritation or inflammation.

We have developed a nano-sized liposomal formulation with a narrow polydispersity, and achieved a high D/L mole ratio of 0.181 using EggPC liposomes. While a similar, high $\mathrm{D} / \mathrm{L}$, mole ratio for other lipophilic drugs such as paclitaxel (0.15), ${ }^{17}$ aryl-imidazole compound (ML220) (0.29), ${ }^{18}$ and curcumin $(0.07)^{19}$ have been reported, the $\mathrm{D} / \mathrm{L}$ mole ratio for other hydrophobic drugs such as ibuprofen $(0.02)^{20}$ and cisplatin (0.05) were low. ${ }^{21}$ We previously achieved a maximum D/L mole ratio of 0.104 using DPPC liposomes, synthesized from a saturated lipid that maintained an IOP lowering effect for 50 days in the rabbit eyes. ${ }^{16}$ Therefore this is a significantly improved formulation in terms of the $\mathrm{D} / \mathrm{L}$ mole ratio using EggPC, with a longer IOP lowering effect in the same species.

Our storage stability analysis showed that liposomes (without latanoprost) aggregated to micron size particles within 6 months of storage at $4^{\circ} \mathrm{C}$. This finding is consistent with previously reported studies with EggPC liposomes. ${ }^{22-24}$ In contrast, notable disintegration of the carrier is usually observed for hydrophobic drug-loaded liposomes, hence limiting its potential for a sustained drug delivery. ${ }^{18,25,26}$ Such instability in size greatly limits the use of liposomal carriers for ocular drug delivery. In our study, the latanoprost-loaded liposome formulation had minimal size changes detected at both $4^{\circ} \mathrm{C}$ ( $>6$ months) and $25^{\circ} \mathrm{C}$ ( $>1$ month), suggesting that latanoprost does not destabilize the bilayer of EggPC liposomes, and may even help to improve the stability under storage conditions, similar to the effects of cholesterol. ${ }^{22,27}$ This is further supported by similar stability of high-loading curcumin into EggPC and paclitaxel into mixed liposomes composed of saturated and unsaturated lipids. ${ }^{19,25}$

As mentioned above, using the partition coefficient values, we find that approximately $95 \%$ of latanoprost is associated in the bilayer of EggPC liposomes, while the remaining $5 \%$ of the drug exists as free drug in the continuous phase, for a $\mathrm{D} / \mathrm{L}$ mole ratio of 0.181 . Because very low 
amounts of free drug was present, no additional step for removal was necessary.

A sustained release of drugs to improve therapeutic efficacy in the management of glaucoma, has now been considered as a major focus in pharmaceutical development for glaucoma medications. Various delivery systems are now being explored. The ideal system should have minimal risks to the patient and be as comfortable as possible to deliver. Liposomes are a potential candidate for providing sustained release of ocular drugs. However, the major limitation with topical liposomes is the lack of sustained efficacy, due to biological barriers in the eye and clearance from tear dilution. ${ }^{7}$ Different routes of injectable liposome systems tested include periocular, intravitreal, and subconjunctival. ${ }^{28,29}$ Moreover, recent reports have shown the benefits of subconjunctival injections with improved absorption rates and sustained efficacy, using low molecular weight heparin, ${ }^{30}$ streptokinase, and clodronate. ${ }^{31,32}$ Here, we show for the first time that the subconjunctival delivery of liposomes can clearly bypass these barriers thereby allowing these nanocarriers to be potentially used as a platform for sustained release of drugs.

Moon et al suggested that the liposomes behaved like a depot system in the subconjunctival space. ${ }^{30}$ They suggested a lack in systemic clearance of liposomes in the subconjunctival space, which remained at the site of injection, probably due to the larger particle size $(\sim 550 \mathrm{~nm})$ of the liposomes. There are several limitations in our pilot study, such as the small numbers and study duration. However in our study, we successfully achieved a sustained reduction in IOP over an extended timeframe of 90 days from a single subconjunctival injection in the rabbit eye. We also demonstrated safety and tolerance of this particular liposomal injection. The initial high burst of the drug observed in the first few days of the in vitro release studies requires further understanding and refinement of the formulation, which we are currently performing. In addition, future studies are planned to track the movement of liposomes in the ocular structures after they have been injected as well as the stability of the latanoprost within the liposomes during this process. Nonetheless, the initial outcomes from our study report are promising and point to significant benefits over the current eyedrop regime for glaucoma.

\section{Conclusion}

In conclusion, we report a liposomal formulation with EggPC to provide sustained release of latanoprost in the rabbit eye for up to 90 days after a single subconjunctival injection. Further detailed studies on the pharmacodynamics of these liposomes within the ocular tissues will determine its future potential clinical application.

\section{Acknowledgment}

The authors would like to acknowledge the financial support provided by the National Research Foundation-Funded Translational and Clinical Research (TCR) Programme Grant [NMRC/TCR/002 - SERI/2008 - TCR 621/41/2008].

\section{Disclosure}

The authors report no conflict of interest in this work.

\section{References}

1. Heijl A, Leske MC, Bengtsson B, Hyman L, Hussein M. Reduction of intraocular pressure and glaucoma progression: results from the Early Manifest Glaucoma Trial. Arch Ophthalmol. 2002;120:1268-1279.

2. Nordmann JP, Auzanneau N, Ricard S, Berdeaux G. Vision related quality of life and topical glaucoma treatment side effects. Health Qual Life Outcomes. 2003;1:75.

3. Kwon YH, Kim YI, Pereira ML, Montague PR, Zimmerman MB, Alward WL. Rate of optic disc cup progression in treated primary open-angle glaucoma. J Glaucoma. 2003;12:409-416.

4. Quigley HA, Tielsch JM, Katz J, Sommer A. Rate of progression in open-angle glaucoma estimated from cross-sectional prevalence of visual field damage. Am J Ophthalmol. 1996;122:355-363.

5. Sakai Y, Yasueda S, Ohtori A. Stability of latanoprost in an ophthalmic lipid emulsion using polyvinyl alcohol. Int J Pharm. 2005;305: 176-179.

6. Linden C, Nuija E, Alm A. Effects on IOP restoration and blood-aqueous barrier after long term treatment with latanoprost in open angle glaucoma and ocular hypertension. Br J Ophthalmol. 1997;81:370-372.

7. Mishra GP, Bagui M, Tamboli V, Mitra AK. Recent applications of liposomes in ophthalmic drug delivery. J Drug Deliv. 2011;2011:863734.

8. Schaeffer HE, Krohn DL. Liposomes in topical drug delivery. Invest Ophthalmol Vis Sci. 1982;22:220-227.

9. Hathout RM, Mansour S, Mortada ND, Guinedi AS. Liposomes as an ocular delivery system for acetazolamide: in vitro and in vivo studies. AAPS Pharm Sci Tech. 2007;8:1. http://www.aapspharmscitech.org/ view.asp?art=pt0801001. Accessed August 23, 2011.

10. Lee VH, Takemoto KA, Iimoto DS. Precorneal factors influencing the ocular distribution of topically applied liposomal inulin. Curr Eye Res. 1984;3:585-591.

11. Hirnle E, Hirnle P, Wright JK. Distribution of liposome-incorporated carboxyfluorescein in rabbit eyes. J Microencapsul. 1991;8:391-399.

12. Hironaka K, Inokuchi Y, Tozuka Y, Shimazawa M, Hara H, Takeuchi H. Design and evaluation of a liposomal delivery system targeting the posterior segment of the eye. J Control Release. 2009;136:247-253.

13. Stewart JC. Colorimetric determination of phospholipids with ammonium ferrothiocyanate. Anal Biochem. 104:10-14.

14. Munger RJ. Veterinary ophthalmology in laboratory animal studies. Vet Ophthalmol. 2002;5:167-175.

15. Amano S, Nakai Y, Ko A, Inoue K, Wakakura M. A case of keratoconus progression associated with the use of topical latanoprost. Jpn J Ophthalmol. 2008;52(4):334-336.

16. Natarajan JV, Chattopadhyay S, Ang M, et al. Sustained release of an anti-glaucoma drug: demonstration of efficacy of a liposomal formulation in the rabbit eye. PLoS ONE. 2011;6:e24513. http://www.plosone. org/article/info\%3Adoi\%2F10.1371\%2Fjournal.pone.0024513. Acessed September 13, 2011.

17. Kan P, Tsao CW, Wang AJ, Su WC, Liang HF. A liposomal formulation able to incorporate a high content of paclitaxel and exert promising anticancer effect. J Drug Deliv. 2011;2011:2-10. 
18. Liu J, Lee H, Huesca M, Young A, Allen C. Liposome formulation of a novel hydrophobic aryl-imidazole compound for anti-cancer therapy. Cancer Chemother Pharmacol. 2006;58:306-318.

19. Pandelidou M, Dimas K, Georgopoulos A, Hatziantoniou S, Demetzos C. Preparation and characterization of lyophilised egg PC liposomes incorporating curcumin and evaluation of its activity against colorectal cancer cell lines. J Nanosci Nanotechnol. 2011;11:1259-1266.

20. Mohammed AR, Weston N, Coombes AG, Fitzgerald M, Perrie Y. Liposome formulation of poorly water soluble drugs: optimisation of drug loading and ESEM analysis of stability. Int J Pharm. 2004;285: 23-34.

21. Perez-Soler R, Khokhar AR. Lipophilic cisplatin analogues entrapped in liposomes: role of intraliposomal drug activation in biological activity. Cancer Res. 1992;52:6341-6347.

22. Drummond DC, Meyer O, Hong K, Kirpotin DB, Papahadjopoulos D. Optimizing liposomes for delivery of chemotherapeutic agents to solid tumors. Pharmacol Rev. 1999;51:691-743.

23. Sarbolouki MN, Toliat T. Storage stability of stabilized MLV and REV liposomes containing sodium methotrexate (aqueous and lyophilized). PDA J Pharm Sci Technol. 1998;52:23-27.

24. Hernandez-Caselles T, Villalain J, Gomez-Fernandez JC. Stability of liposomes on long term storage. J Pharm Pharmacol. 1990;42: 397-400.

25. Bernsdorff C, Reszka R, Winter R. Interaction of the anticancer agent Taxol (paclitaxel) with phospholipid bilayers. J Biomed Mater Res. 1999;46:141-149.
26. Khan DR, Rezler EM, Lauer-Fields J, Fields GB. Effects of drug hydrophobicity on liposomal stability. Chem Biol Drug Des. 2008;71: 3-7.

27. Zhang B, Lu Y, Chen J, Wu W. Effects of interior gelation on pharmacokinetics and biodistribution of liposomes encapsulating an anti-cancer drug cytarabine. J Biomed Nanotechnol. 2010;6:704-709.

28. Assil KK, Frucht-Perry J, Ziegler E, Schanzlin DJ, Schneiderman T, Weinreb RN. Tobramycin liposomes. Single subconjunctival therapy of pseudomonal keratitis. Invest Ophthalmol Vis Sci. 1991;32: 3216-3220.

29. Peyman GA, Schulman JA, Khoobehi B, Alkan HM, Tawakol ME, Mani H. Toxicity and clearance of a combination of liposome-encapsulated ganciclovir and trifluridine. Retina. 1989;9:232-236.

30. Moon JW, Song YK, Jee JP, Kim CK, Choung HK, Hwang JM. Effect of subconjunctivally injected, liposome-bound, low-molecular-weight heparin on the absorption rate of subconjunctival hemorrhage in rabbits. Invest Ophthalmol Vis Sci. 2006;47:3968-3974.

31. Baek SH, Park SJ, Jin SE, Kim JK, Kim CK, Hwang JM. Subconjunctivally injected, liposome-encapsulated streptokinase enhances the absorption rate of subconjunctival hemorrhages in rabbits. Eur J Pharm Biopharm. 2009;72:546-551.

32. Fukushima A, Ozaki A, Ishida W, van Rooijen N, Fukata K, Ueno H. Suppression of macrophage infiltration into the conjunctiva by clodronate liposomes in experimental immune-mediated blepharoconjunctivitis Cell Biol Int. 2005;29:277-286.
International Journal of Nanomedicine

\section{Publish your work in this journal}

The International Journal of Nanomedicine is an international, peerreviewed journal focusing on the application of nanotechnology in diagnostics, therapeutics, and drug delivery systems throughou the biomedical field. This journal is indexed on PubMed Central,

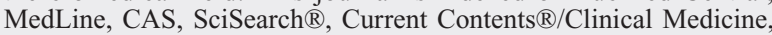

\section{Dovepress}

Journal Citation Reports/Science Edition, EMBase, Scopus and the Elsevier Bibliographic databases. The manuscript management system is completely online and includes a very quick and fair peer-review system, which is all easy to use. Visit http://www.dovepress.com/ testimonials.php to read real quotes from published authors. 